\title{
Mapping background values of atmospheric nitrogen total depositions in Germany based on EMEP deposition modelling and the European Moss Survey 2005
}

\section{Kartierung der Hintergrundwerte atmosphärischer Stickstoff-Gesamtdepositionen in Deutschland anhand von Daten des EMEP-Messnetzes und des ICP Vegetation Moos-Monitoring 2005}

Winfried Schröder ${ }^{1+}$, Marcel Holy ${ }^{1+}$, Roland Pesch ${ }^{* 1+}$, Harry Harmens ${ }^{2}$ and Hilde Fagerli ${ }^{3}$

\begin{abstract}
Background In order to map exceedances of critical atmospheric deposition loads for nitrogen ( $\mathrm{N}$ ) surface data on the atmospheric deposition of $\mathrm{N}$ compounds to terrestrial ecosystems are needed. Across Europe such information is provided by the international European Monitoring and Evaluation Programme (EMEP) in a resolution of $50 \mathrm{~km}$ by $50 \mathrm{~km}$, relying on both emission data and measurement data on atmospheric depositions. The objective of the article at hand is on the improvement of the spatial resolution of the EMEP maps by combining them with data on the $\mathrm{N}$ concentration in mosses provided by the International Cooperative Programme on Effects of Air Pollution on Natural Vegetation and Crops (ICP Vegetation) of the United Nations Economic Commission for Europe (UNECE) Long-range Transboundary Air Pollution (LTRAP) Convention.

Methods The map on atmospheric depositions of total $\mathrm{N}$ as modelled by EMEP was intersected with geostatistical surface estimations on the $\mathrm{N}$ concentration in mosses at a resolution of $5 \mathrm{~km}$ by $5 \mathrm{~km}$. The medians of the $\mathrm{N}$ estimations in mosses were then calculated for each $50 \mathrm{~km}$ by $50 \mathrm{~km}$ grid cell. Both medians of moss estimations and corresponding modelled deposition values were In-transformed and their relationship investigated and modelled by linear regression analysis. The regression equations were applied on the moss kriging estimates of the $\mathrm{N}$ concentration in mosses. The respective residuals were projected onto the centres of the EMEP grid cells and were mapped using variogram analysis and kriging procedures. Finally, the residual and the regression map were summed up to the map of total $\mathrm{N}$ deposition in terrestrial ecosystems throughout Europe.

Results and discussion The regression analysis of the estimated $\mathrm{N}$ concentrations in mosses and the modelled EMEP depositions resulted in clear linear regression patterns with coefficients of determination of $r^{2}=0.62$ and Pearson correlations of $r_{p}=0.79$ and Spearman correlations of $r_{s}=0.70$, respectively. Regarding the German territory a nationwide mean of $18.1 \mathrm{~kg} / \mathrm{ha} / \mathrm{a}$ (standard deviation: $3.49 \mathrm{~kg} / \mathrm{ha} / \mathrm{a}$ ) could be derived from the resulting map
\end{abstract}

\footnotetext{
†These authors contributed equally to this work

*Correspondence: rpesch@iuw.uni-vechta.de

${ }^{1}$ Chair of Landscape Ecology, University of Vechta, P.O.B. 1553, 49364 Vechta,

Germany

Full list of author information is available at the end of the article
}

\section{SpringerOpen ${ }^{\odot}$}

(c) 2011 Schröder et al; licensee Springer. This is an open access article distributed under the terms of the Creative Commons Attribution License (http://creativecommons.org/licenses/by/2.0), which permits unrestricted use, distribution, and reproduction in any medium, provided the original work is properly cited. 
on total $\mathrm{N}$ deposition in a resolution of $5 \mathrm{~km}$ by $5 \mathrm{~km}$. Recent updates of the modelled atmospheric deposition of $\mathrm{N}$ provided a similar estimate for Germany.

Conclusions The linking of modelled EMEP data on the atmospheric depositions of total $\mathrm{N}$ and the accumulation of $\mathrm{N}$ in mosses allows to map the deposition of total $\mathrm{N}$ in a high resolution of $5 \mathrm{~km}$ by $5 \mathrm{~km}$ using empirical moss data. The mapping relies on the strong statistical relationship between both processes that are physically and chemically related to each other. The mapping approach thereby relies on available data that are both based on European wide harmonized methodologies. From an ecotoxicological point of view the linking of data on $\mathrm{N}$ depositions and those on $\mathrm{N}$ bioaccumulation can be considered a substantial progress.

Keywords EMEP; moss surveys; ICP Vegetation; atmospheric nitrogen depositions; biomonitoring; modelling

\section{Zusammenfassung}

Hintergrund Für die Kartierung kritischer Eintragsraten (Critical Loads, CL) für Stickstoff (N) werden flächendeckende Depositionsdaten benötigt. Diese werden europaweit im EMEP-Programm und auf nationalstaatlicher Ebene in Forschungsprojekten zur Verfügung gestellt. Es handelt sich um Ergebnisse aus Modellierungen, die u.a. auf Messwerten der N-Emissionen und der atmosphärischen N-Deposition beruhen. Dieser Artikel stellt am Beispiel der Daten zur N-Deposition aus dem European Monitoring and Evaluation Programme (EMEP) dar, wie deren räumliche Auflösung durch Kombination mit Daten der N-Anreicherung in Moosen aus dem International Cooperative Programme on Effects of Air Pollution on Natural Vegetation and Crops (ICP Vegetation) der United Nations Economic Commission for Europe (UNECE) Long-range Transboundary Air Pollution (LTRAP) Convention.erhöht werden kann.

Methoden Die in einer Auflösung von $50 \mathrm{~km}$ mal $50 \mathrm{~km}$ vorliegende EMEP N-Depositionskarte wurde mit geostatistich validen Kriging-Karten über die Anreicherung von N in Moosen in einem Geografischen Informationssystem (GIS) verknüpft. Anschließend wurden die Mediane aller $5 \mathrm{~km}$ mal $5 \mathrm{~km}$ großen Rasterzellen der $\mathrm{N}$-Anreicherungskarte innerhalb der jeweiligen $50 \mathrm{~km}$ mal $50 \mathrm{~km}$ abdeckenden EMEP-Rasterzellen berechnet. Die Mediane der geschätzten Elementkonzentrationen im Moos sowie die Depositionswerte wurden In-transformiert und korrelations- und regressionsanalytisch untersucht. Sodann wurden die Regressionsfunktionen auf die Kriging-Flächenkarten der N-Anreicherungen in Moosen angewendet. Die Residuen der Regressionsfunktion wurden bestimmt, entlogarithmiert, auf die Mittelpunkte der entsprechenden EMEP-Rasterzellen projiziert, variogrammanalytisch auf räumliche Strukturen untersucht und mit Lognormal-Kriging flächenhaft interpoliert. Die Kriging-Karte der Residuen wurde abschließend mit der regressionsanalytisch berechneten N-Depositionsflächenkarte verrechnet.

Ergebnisse und Diskussion Die Regressionsanalyse zeigt, dass die N-Anreicherung in den Moosen aus Hintergrundgebieten mit der N-Gesamtdeposition europaweit mit Pearson Korrelationen von $r_{p}=0.79$ sowie Spearman Korrelationen von $r_{s}=0.70$ korreliert ist. Das Bestimmtheitsmaß des Regressionsmodells beträgt $r^{2}=0,62$. Die statistische Auswertung der auf dieser Grundlage berechneten Karte der N-Gesamtdeposition ergibt einen deutschlandweiten Mittelwert der von $18.1 \mathrm{~kg} / \mathrm{ha} / \mathrm{a}$ (Standardabweichung $3.49 \mathrm{~kg} / \mathrm{ha}$ / a). Vergleicht man die Ergebnisse dieser Berechnungen mit Ergebnissen aus anderen Verfahren, so zeigen sich z.T. Unterschiede. Die am Ende des Jahres 2009 anlässlich eines Workshops zur Modellierung von Schadstoffeinträgen und ihren Wirkungen auf Ökosysteme veröffentlichten N-Gesamtdepositionsmodellierungen entsprechen allerdings ungefähr denen, die anhand der Daten aus dem EMEP und ICP Vegetation in dieser Untersuchung berechnet wurden.

Schlussfolgerungen Die Verknüpfung der Daten zur N-Gesamtdeposition (EMEP) und der N-Anreicherungen in Moosen (ICP Vegetation) ermöglicht eine empirisch validierte, räumlich differenzierte Kartierung der $\mathrm{N}$-Gesamtdeposition. Die ausgeprägte, statistisch hoch signifikante Korrelation zwischen den beiden physikalisch und chemisch miteinander verbundenen Prozessen der atmosphärischen Deposition und der Bioakkumulation bilden die Grundlage der Kartierung. Die Karten nutzen vorhandenes Datenmaterial, das auf der Grundlage europaweit harmonisierter Methoden in zwei qualitätskontrollierten Messprogrammen erhoben wurde. Aus dem Blickwinkel der Ökotoxikologie ist die Verknüpfung von Daten über Stoffeinträge in terrestrische Ökosysteme und N-Anreicherungen in deren Moosbiomasse ein Fortschritt.

Schlagwörter EMEP; Moos-Monitoring; ICP Vegetation; Stickstoffdepositionen; Biomonitoring; Modellierung 


\section{Hintergrund}

Landwirtschaft, industrielle Produktion, Verkehr und Waldbrände sind bedeutende Emissionsquellen für $\mathrm{N}$ haltige Verbindungen in der Atmosphäre [1,2]. Die oxidierten (NOx) und reduzierten (NHy) N-Komponenten gelangen nach bis zu 2000 km bzw. bis zu $500 \mathrm{~km}$ Transport mit Regen und Schnee als nasse Depositionen, mit Wolken bzw. Nebel als okkulte (feuchte) Deposition und partikulär oder gasförmig als trockene Deposition an die Erdoberfläche [3]. Solche N-Einträge können die Eutrophierung und Versauerung von terrestrischen und aquatischen Ökosystemen sowie Veränderungen ihrer Biodiversität nach sich ziehen [4-8]. Umweltpolitische Maßnahmen zur Reduktion der N-Emissionen sind in Deutschland beispielsweise die Biodiversitätsstrategie und das Stickstoffminderungsprogramm und auf europäischer Ebene die Genfer Luftreinhaltekonvention (Convention on Long-range Transboundary Air Pollution, LRTAP). Neben der Überwachung der N-Emissionen und -Einträge muss die N-Anreicherung in der Umwelt räumlich differenzierend erfasst werden [9].

EMEP erfüllt für die LRTAP-Convention u.a. folgende Aufgaben: Erhebung von Emissionsdaten, Messung von Konzentrationen ausgewählter Stoffe in der Luft und in Niederschlägen (EMEP Chemical Coordinating Centre, EMEP-CCC) sowie Modelling des atmosphärischen Transports und der Deposition dieser Stoffe. Die Speicherung und Verteilung dieser Daten erfolgt durch das Centre on Emission Inventories and Projections (CEIP). Die Modellierung der Schwefel- und N-Verbindungen sowie der Photooxidantien erfolgt im Meteorological Synthesizing Centre West (MSC-W, Oslo), die Modellierung des Transports und der Deposition der Schwermetalle Blei, Cadmium und Quecksilber im Meteorological Synthesizing Centre East (MSC-ER, Moskau). Die Depositionsmessungen erfolgen je nach Komponente europaweit an bis zu 70 Orten, acht davon entfielen im Jahr 2000 auf Deutschland [10]. Die versauernden und eutrophierenden Depositionen sowie Ozon werden mit dem EMEP MSC-W Unified Eulerian Chemical Transport Model mit einer räumlichen Auflösung von $50 \mathrm{~km} \times 50 \mathrm{~km}$ berechnet [11].

Die Modellergebnisse werden anhand der Messdaten aus dem EMEP-Netz validiert. Zusätzlich wird versucht, andere geeignete Daten zu nutzen. Dabei stellte sich heraus, dass aus sieben europäischen Ländern 160 der 860 Level II-Standorte des International Co-operative Programme on Assessment and Monitoring of Air Pollution Effects on Forests (ICP Forests) die von [10] herangezogenen Qualitätskriterien erfüllten und zur Validierung der N-Gesmtdeposition genutzt werden konnten. 26 der 89 deutschen ICP Forests Level IIStandorten lieferten zur Validierung der EMEP-Modellierungen brauchbare Daten. Ein Problem bestand auch in der mangelnden Repräsentativität der ICP Forests Level II-Standorte für die Depositionsmodellierung [10]. Insbesondere mit Blick auf die Berechnung von CL wird die Einbeziehung weiterer Daten angestrebt, um zu einer höheren räumlichen Auflösung der Depositionsmodellierung zu gelangen. Ein Schritt, dieses Ziel zu erreichen, ist die Einbeziehung von Daten aus einem räumlich Dichten Messnetz, in dem ein Phänomen erfasst wird, das physikalisch eng mit der Deposition verknüpft ist: die Stoffanreicherung an der Erdoberfläche.

Im ICP Vegetation werden Anreicherungen von Schwermetallen seit 1990 alle fünf Jahre und Stickstoff seit 2005 an bis zu 7000 bzw. rund 3000 Orten in bis zu 30 bzw. 16 europäischen Staaten erfasst [12-14]. Die gesammelten Moose haben sich zum Monitoring von Schwermetallanreicherung, mittlerweile aber auch zur Erfassung der N-Akkumulation bewährt [15-21]. Die Anreicherung von toxischen, eutrophierenden oder versauernden Stoffen ist ein wichtiger Gesichtspunkt der ökotoxikologischen Bewertung stofflicher Wirkungen.

Depositionsdaten erhalten einen Wirkungsbezug, wenn sie bei der Berechnung von Critical Loads (CL) [22,23] als Predicted Environmental Concentration (PEC) mit Predicted No Effect Concentrations (PNEC) von Ökosystemen verknüpft werden [24]. Die Aussagekraft von CL bemisst sich nach der Qualität des Modells, d.h. von seiner intrinsischen (Un)Sicherheit, der Qualität der Eingangsdaten (Emissionsdaten und Depositionsdaten) $[25,26]$ und ihrer räumlichen Auflösung [23,27]. Die am Ende des Jahres 2009 anlässlich eines Workshops zur Modellierung von Schadstoffeinträgen und ihren Wirkungen auf Ökosysteme veröffentlichten Ergebnisse der N-Gesamtdepositionsmodellierungen liegen um durchschnittlich $35 \%$ niedriger als die zuletzt von $[28,29]$, bei den Schwermetallen Blei und Cadmium betragen die Unterschiede mindestens $200 \%$ [30]. Um die räumliche Validität und damit die Aussagekraft von Depositionsmodellierungen empirisch zu untermauern, liegt es nahe, die statistischen Beziehungen zwischen Depositionsdaten und Daten aus dem räumlich dichten Messnetz des Europäischen Moos-Monitoring regressionsanalytisch $\mathrm{zu}$ quantifizieren und die Regressionsmodelle auf die Messpunkte des Moosmessnetzes oder die daraus abgeleiteten flächendeckenden und räumlich hoch aufgelösten Karten der N-Akkumulation in Moosen anzuwenden. Ziel dieses Artikels ist es, die dazu angewendete Methodik und die damit erzielten Ergebnisse für die N-Gesamtdeposition im Jahr 2005 für das Gebiet der Bundesrepublik Deutschland darzustellen.

\section{Methoden \\ Moos-Monitoring}

Ziel des ICP Vegetation Moos-Monitoring ist es, die großräumigen Muster und zeitlichen Entwicklungen der 
Bioakkumulation von Schwermetallen (seit 1990 alle fünf Jahre) und Stickstoff (seit 2005/6) Staaten übergreifend zu kartieren. Dabei geht es in erster Linie um die Erfassung darum, sogenannte Hintergrundwerte $\mathrm{zu}$ erhalten und nicht darum, emittentenbezogene Aussagen zu gewinnen. Von potenziellen Emissionsquellen sind bestimmte Abstände einzuhalten, und an Orten mit sichtbaren technischen Emissionsquellen in der Umgebung werden keine Moosproben entnommen. In der Messkampagne 2005/6 wurden an 2781 Orten in Belgien, Bulgarien, Estland, Finnland, Frankreich, Deutschland, Großbritannien, Italien (Region Bolzano), Lettland, Österreich, Slovakei, Slovenien, Spanien (Navarra), Schweiz, der Teschechischen Republik sowie der Türkei Moosproben nach einer Richtlinie [31] entnommen und auf Stickstoff untersucht. Hierbei kamen auf $1000 \mathrm{~km}^{2}$ mindestens 1,5 Moosentnahmestellen. In Deutschland führten Bund und Länder das Moos-Monitoring in den Jahren 1990, 1995, 2000 und 2005 gemeinsam durch. Die räumliche Dichte des Moosmessnetzes betrug mit 1,7 (1990), 2,9 (1995, 2000) und 2,0 (2005) Standorte pro $1000 \mathrm{~km}^{2}$. Die Messnetzausdünnung beim deutschen Monitoring in 2005 erfolgte ohne Einbußen der geostatistischen Validität und Landschaftsrepräsentanz des Messnetzes [32].

Von den an 2781 Orten Europas gesammelten Moosproben für N-Analysen entfielen 43,4 \% auf Pleurozium schreberi, 20,0 \% auf Hylocomium splendens, 19,0 \% auf Hypnum cupressiforme, 11,1 \% auf Pseudoscleropodium purum und 6,5\% auf weitere Arten. Die Moosproben wurden bei $40^{\circ} \mathrm{C}$ getrocknet, und die N-Gehalte nach der Methode von [33] oder mit der Elementaranalyse nach [34] quantitativ als \%-Anteile der Moostrockenmasse bestimmt. Die Qualität der Messungen und Probenentnahmen wurde umfassend im Sinne von [35] gesichert und dokumentiert $[12,13,24,36]$. Die Qualitätskontrolle der Messdaten erfolgte anhand der Referenzmaterialien M2 und M3 [37]. Zusätzlich wurden zertifizierte Referenzmaterialien verwendet. Die Ergebnisse wiesen auf eine gute Übereinstimmung zwischen den beteiligten Laboratorien, den analytischen Verfahren und den Zielwerten der Referenzmaterialien hin $[12,13]$. Die Daten wurden im Programmzentrum des ICP Vegetation auf Plausibilität und Extremwerte untersucht. Die ggf. bereinigten Datensätze und erste Karten über die geographischen Muster der N-Gehalte in den Moosen wurden den ExpertInnen in den Teilnehmerstaaten zur Endkontrolle übergeben. Die räumlichen Muster der N-Gehalte in den Moosen wurden mit den EMEP-Daten über die N-Deposition (Abschnitt Depositionsmodellierung) regressionsanalytisch verknüpft (Abschnitt Berechnung der Depositionskarten).

\section{Depositionsmodellierung}

Das EMEP-Modell zur Berechnung der N-Deposition wurde im Norwegischen Institut für Meteorologie (Olso) entwickelt. Es basiert auf früheren Modellentwicklungen $[38,39]$ und wird umfassend von [40] und [41] erläutert. Die Validierungen der Modellergebnisse sind umfassend dargestellt [10,11,42-47]. Das EMEP Unified Eulerian Chemical Transport Model ist ein Mehrebenen Transportund Depositionsmodell. Der von ihm geographisch abgedeckte Teil der Erdoberfläche, die EMEP model domain, umfasst Europa, den Nordatlantik und die Polarregion [40,41]. Das Modell ist auf 20 vertikale Layer und eine horizontale Auflösung von $50 \mathrm{~km} \times 50 \mathrm{~km}$ (in $60^{\circ} \mathrm{N}$ ) ausgelegt. Die Daten zu anthropogenen Emissionen sind nach Sektoren und Rasterzellen entsprechend der offiziellen Datenlieferungen im Rahmen der LRTAP Convention differenziert [48]. Das Modell berechnet rund 140 chemische Reaktionen zwischen 70 chemischen Komponenten.

\section{Berechnung der Depositionskarten}

Die Kartierung der N-Gehalte in Moosen erfolgte mit den geostatistischen Methoden Variogrammanalyse und Kriging-Interpolation. Die Qualität dieser Flächenschätzungen wurde durch Kreuzvalidierung ermittelt [24]. Sodann wurden die EMEP-Daten zur N-Gesamtdeposition 2005 mit der europaweit geostatistisch geschätzten Flächenkarte der N-Konzentration in den 2005 gesammelten Moosen verschnitten. Anschließend wurden die Mediane aller $5 \mathrm{~km}$ mal $5 \mathrm{~km}$ großen Rasterzellen der N-Anreicherungskarte innerhalb der jeweiligen $50 \mathrm{~km}$ mal $50 \mathrm{~km}$ EMEP-Rasterzellen berechnet. Die Mediane der geschätzten Elementkonzentrationen im Moos sowie die Depositionswerte wurden ln-transformiert und korrelations- und regressionsanalytisch untersucht. Sodann wurden die Regressionsfunktionen auf die Kriging-Flächenkarten der NAnreicherungen in Moosen angewendet. Die Residuen der Regressionsfunktion wurden bestimmt, entlogarithmiert, auf die Mittelpunkte der entsprechenden EMEP-Rasterzellen projiziert, variogrammanalytisch auf räumliche Strukturen untersucht und mit LognormalKriging flächenhaft interpoliert. Die Kriging-Karte der Residuen wurde abschließend mit der regressionsanalytisch berechneten $\mathrm{N}$-Depositionsflächenkarte verrechnet, so dass die durch die beiden lineare Regressionsmodelle bedingten räumlich differenzierten Unter- bzw. Überschätzungstendenzen minimiert wurden. Aus dieser Europakarte der N-Gesamtdeposition des Jahres 2005 wurde das Gebiet der Bundesrepublik Deutschland ausgestanzt. Diese Deutschlandkarte zeigt die räumliche Differenzierung der N-Gesamtdeposition als Vielfache der jeweiligen Standardabweichung vom Bundesdurchschnitt. Die dabei gewählte räumliche Auflösung orientiert sich an der Standardabweichung der Nachbarschaftsdistanzen der Beprobungspunkte in Europa und beträgt dementsprechend $5 \mathrm{~km}$ mal $5 \mathrm{~km}$. 


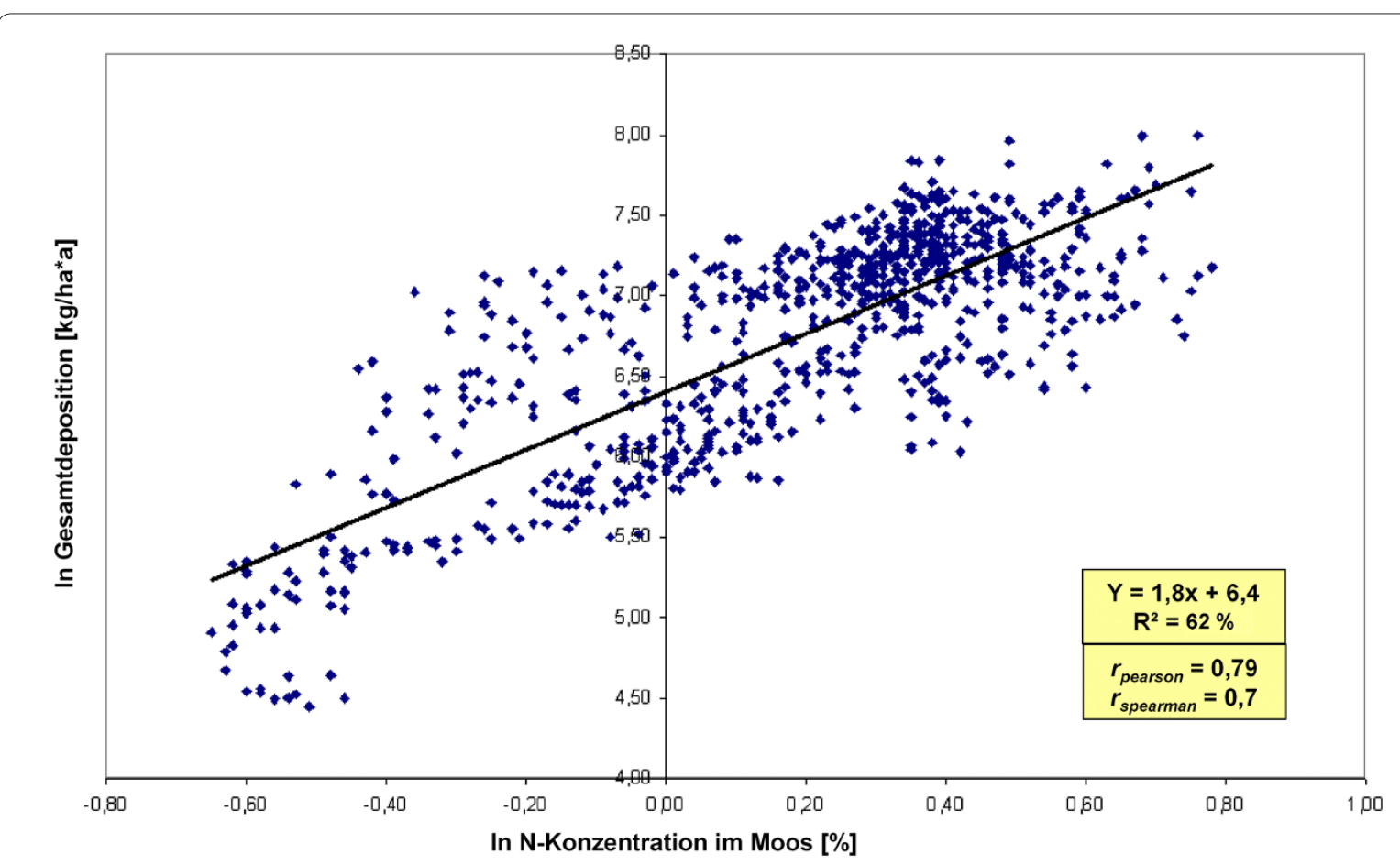

Abb. 1. Regressionsmodell für die Beziehung zwischen N-Gehalten in Moosen (ICP Vegetation Moss Survey 2005) und modellierter $\mathrm{N}$-Gesamtdeposition (EMEP 2005).

\section{Ergebnisse}

Für die Validierung der EMEP-Modellierung lagen an den EMEP Messstationen nur Messungen zur nassen Deposition vor. Diesbezüglich wurde eine Abweichung der qualitätskontrollierten EMEP-Messwerte von den Modellierungen um ca. 30 \% berechnet. Die Qualität der Messungen der N-Konzentrationen in den Moosen wurde anhand von Referenzmaterial kontrolliert. Die aus diesen Messwerten des Moos-Monitoring mit KrigingInterpolation berechnete Europa-Karte der N-Anreicherung in Moosen gibt ausgeprägte räumliche Strukturen wieder und weist eine ausreichende Schätzqualitäten auf: Der mittlere (Median) korrigierte prozentuale Fehler beträgt 9,23 \%, der Pearson Korrelationskoeffizient zwischen Mess- und Schätzwerten beläuft sich auf 0,72 für das Nugget / Sill-Verhältnis des der Schätzung zugrundeliegenden Variogramm-Modells wurde ein Wert von $64 \%$, und für die Aussagereichweite der punktuellen Messungen $312 \mathrm{~km}$ berechnet.

Die statistischen Beziehungen zwischen den NKonzentrationen in Moosen und modellierter N-Gesamtdeposition wurden nach Verknüpfung der entsprechenden Karten im GIS regressionsanalytisch quantifiziert (Abb. 1). Korrelationsanalysen zeigen, dass die NAnreicherung in den Moosen mit der N-Gesamtdeposition europaweit mit $r p=0,79$ (Korrelation nach Pearson) bzw. $r s=0,70$ (Korrelation nach Spearman) korreliert ist. Das Bestimmtheitsmaß des Regressionsmodells beträgt $r^{2}=0,62$. Die statistische Auswertung der auf dieser Grundlage berechneten Karte der N-Gesamtdeposition ergibt einen deutschlandweiten Mittelwert der von 18,1 kg/ ha / a (Standardabweichung 3,49 kg / ha / a). Die Karte zeigt die räumliche Differenzierung der NGesamtdeposition als Vielfache der Standardabweichung. Die dabei gewählte räumliche Auflösung orientiert sich an der Standardabweichung der Distanzen zwischen je zwei Beprobungspunkten innerhalb von Europa und beträgt dementsprechend $5 \mathrm{~km}$ mal $5 \mathrm{~km}$ (Abb. 1).

Die Residuen, also der nicht 'erklärte' Anteil der in dem linearen Regressionsmodell quantifizierten Beziehung zwischen modellierter N-Gesamtdeposition und $\mathrm{N}$ Bioakkumulation wurden variogrammanalytisch auf räumliche Strukturen untersucht. Das resultierende Variogramm (Abb. 2) zeigt eine deutliche Autokorrelation der Residuen: Der Betrag der Semivarianz Betrag $(\gamma)$ wächst mit zunehmender Distanz (h) der Moossammelorte voneinander an, und das Nugget / Sill-Verhältnis beträgt 12,8. Diese von den Regressionsmodellen nicht erfasste und in der regressionsanalytisch erzeugten Karte der N-Gesamtdeposition nicht abgebildete Information über die räumliche Struktur der Daten wird im nächsten Schritt dadurch eingebunden, dass anhand der Residuen-Variogramme eine Kriging-Karte berechnet wurde, die mit der 


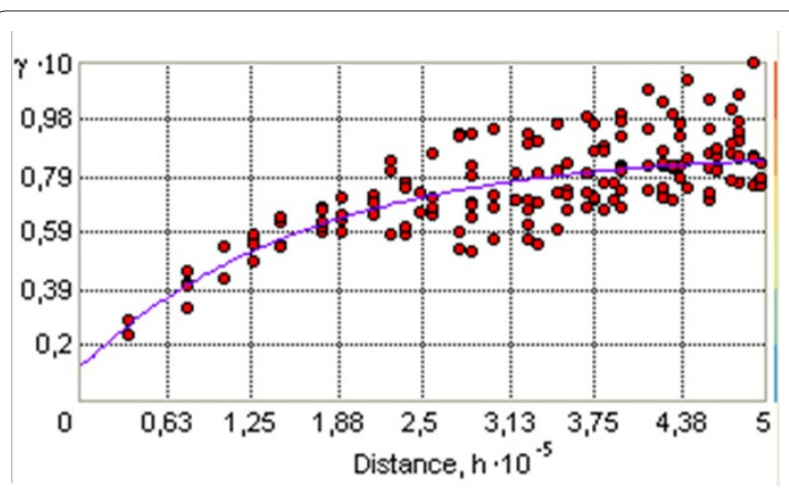

Abb. 2. Variogramm der Residuen des Regressionsmodells für die Beziehung zwischen N-Gehalten in Moosen (ICP Vegetation Moss Survey 2005) und modellierter N-Gesamtdeposition (EMEP 2005).

regressionsanalytisch erzeugten N-Gesamtdepositionskarte durch Addition zu einer GIS-Karte zusammengefügt wurde. Aus dieser Karte wurde das Gebiet der Bundesrepublik Deutschland ausgestanzt (Abb. 3).

Tabelle 1 enthält statistische Maßzahlen, welche die Ergebnisse der Kartierung der N-Deposition zusammenfassend beschreiben. Zusammen mit der aus den Karte ersichtlichen räumlichen Differenzierungen der $\mathrm{N}$ Gesamtdepositionen ergibt sich folgendes Bild: Die minimalen Hintergrundwerte der N-Gesamtdeposition betragen 9,6 kg / ha / a, die maximalen $29,4 \mathrm{~kg} / \mathrm{ha} / \mathrm{a}$. Räumliche Schwerpunkte oberhalb des bundesweiten arithmetischen Mittelwertes der Hintergrundwerte der N-Deposition von $18,1 \mathrm{~kg} / \mathrm{ha} / \mathrm{a}$ sind im Wesentlichen in Nord- und Nordwestdeutschland, den westlichen Teil Mecklenburg-Vorpommerns sowie in Süddeutschland zu erkennen. Unterdurchschnittliche N-Einträge werden für das Saarland, Teile Hessens sowie die meistern Regionen der ehemaligen DDR mit Ausnahme einer Region im östlichen Mecklenburg-Vorpommern und Sachsen berechnet.

\section{Diskussion}

Vergleicht man die Ergebnisse dieser Berechnungen mit denen aus anderen Verfahren, so zeigt sich, dass die nach dem vorgestellten Ansatz kalkulierten N-Gesamtdepositionsmittelwerte für Hintergrundgebiete $(18,1 \mathrm{~kg} /$ ha / a) um rund $34 \%$ niedriger ist als die von $[28,29]$ berechnen 27,9 kg / ha / a. Die am Ende des Jahres 2009 anlässlich eines Workshops zur Modellierung von Schadstoffeinträgen und ihren Wirkungen auf Ökosysteme veröffentlichten $\mathrm{N}$-Gesamtdepositionsmodellierungen liegen um durchschnittlich $35 \%$ niedriger als die oben genannten Berechnungsergebnisse [30]. Damit entsprechen sie ungefähr denen, die anhand der Daten aus dem EMEP und ICP Vegetation in dieser Untersuchung berechnet wurden. Die Aussagekraft der in dieser

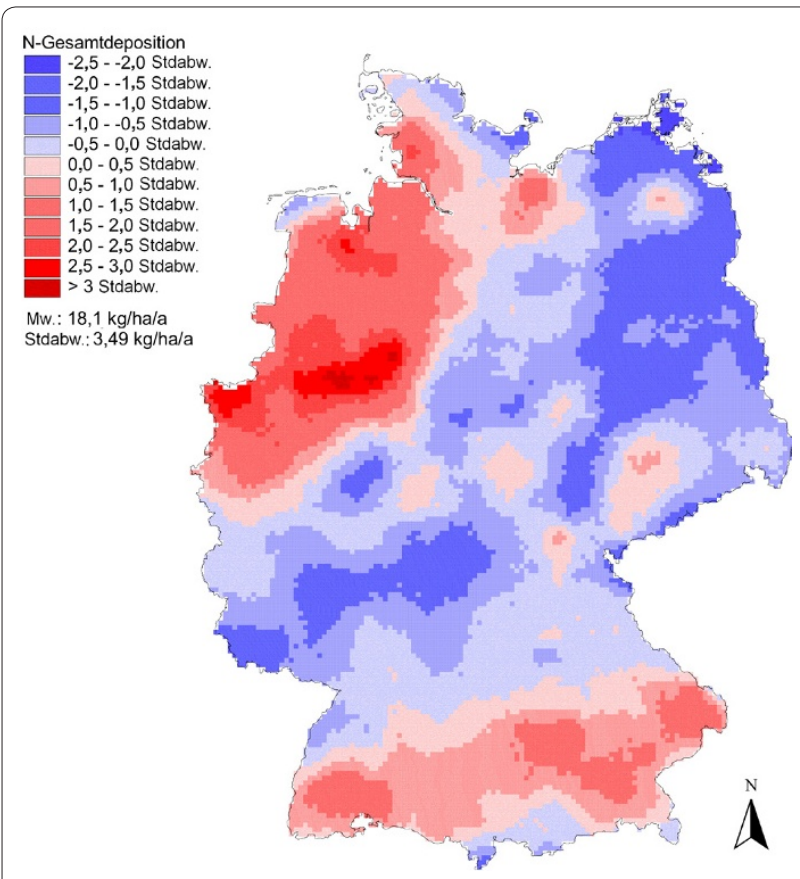

Abb. 3. Regression-Kriging-Flächenschätzung der $\mathrm{N}$-Gesamtdeposition in Deutschland (2005) auf Grundlage von $\mathrm{N}$-Gehalten in Moosen (ICP Vegetation Moss Survey 2005) und modellierter N-Gesamtdeposition (EMEP 2005), Auflösung 5 km mal 5 km.

Tabelle 1. Deskriptiv-statistische Maßzahlen der mit Regression-Kriging aus EMEP-Depositionsdaten und Akkumulationsdaten aus dem Europäischen MoosMonitoring berechneten Karte der N-Gesamtdeposition

\begin{tabular}{lc}
\hline Statistische Maßzahl & $\mathbf{N}$ [kg / ha / a] \\
\hline Minimum & 9,6 \\
Maximum & 29,4 \\
Arithmetischer Mittelwert & 18,1 \\
Standardabweichung & 3,5 \\
20. Perzentil & 15,1 \\
50. Perzentil & 17,3 \\
90. Perzentil & 23,3 \\
98. Perzentil & 26,3 \\
\hline
\end{tabular}

Untersuchung berechneten N-Depositionskarte sollte auch durch einen Abgleich mit Messdaten des in Deutschland betriebenen Depositionsmonitoring geprüft werden. Diese Daten waren im Rahmen der beiden letzten Moos-Monitoringprojekte weder über die Messnetzbetreiber noch über das Umweltbundesamt verfügbar, obwohl eine umfangreiche, die Depositionsdaten der Bundesländer umfassende Datenbank angelegt und in mehreren Forschungsvorhaben fortgeschrieben wurde $[28,29,49-53]$. In den genannten Forschungsberichten werden Schwierigkeiten beschrieben, die bei der 
Zusammenführung der Daten aus mehreren Messnetzen und hinsichtlich ihrer Qualität bestehen. [49] berichtet über die Notwendigkeit, Daten aus Qualitätsgründen auszuschließen, was zur räumlichen Ausdünnung der Daten für geostatistische Modellierungen führt. Weitere Qualitätsprobleme, mit denen sich die Depositionsmodellierung auseinanderzusetzen hat, zeigen [49-53] eindrücklich auf.

Diese Probleme decken sich mit Erfahrungen, die auf europäischer Ebene bei der Auswertung und Modellierung von Depositionen auftreten: Von 860 ICP Forests Level II-Flächen standen [22] für Auswertungen Depositionsdaten (bulk, throughfall) 185 bzw. 249 mit maximal 30 fehlenden Messungen pro Jahr im Zeitraum 2000 bis 2005 zur Verfügung. [10] mussten Daten von 160 ICP-Forests Monitoring-Standorten in sieben europäischen Ländern von einer Untersuchung ausschließen, in der die im EMEP modellierten Depositionen anhand von Messwerten überprüft werden sollten. Dabei erfüllten von 89 deutschen ICP Level II-Standorten 26 die Qualitätsanforderungen der EMEP-Modellierer. Qualitätssicherung, die Harmonisierung der Messprogramme hinsichtlich räumlicher und zeitlicher Kriterien sowie die Methodenstandardisierung sind weiterhin Herausforderungen des Forst-Monitoring [54].

Die EMEP-Modellierer zogen aus diesen Problemen andere Konsequenzen als diejenigen Experten und Behörden, die in Deutschland mit der Depositionsmodellierung befasst sind: Es wurde vereinbart, die im Rahmen ICP Vegetation an bis zu 7000 Orten in Europa erhobenen Daten über die Schwermetall- und Stickstoffgehalte in Moosen mit den im EMEP modellierten Gesamtdepositionen zu verknüpfen und die statistischen Beziehungen zwischen den Datensätzen daraufhin zu überprüfen, ob die Moosdaten als statistische Hilfsvariable für die Berechnung von räumlich hoch aufgelösten Depositionskarten sein können, so wie dies in ähnlichen Zusammenhängen auch erfolgt [55-59]. Damit macht sich EMEP die Vorteile des MoosMonitoring zunutze. Diese sind darin zu sehen, dass das experimentelle Design etwa im Vergleich zum ForstMonitoring sehr viel weniger komplex angelegt ist und die Qualitätskontrolle vom Programmstart an von der Probenentnahme bis zur Datenauswertung reichte, also integraler Bestandteil des Monitoring-Konzeptes war. Damit ist eine zentrale Forderung von [25] an Umweltmonitoring-Programme erfüllt. ICP Forest bereitet die Qualitätssicherung insbesondere bei den Probeneinsammlungen und bei der Datenzusammenführung und Auswertung noch immer Probleme [26]. [60] weisen darauf hin, dass mit dem Moos-Monitoring ein höherer Anteil der Gesamtdeposition als über wetonly- und Bulk-Sammler gemessen werden kann. Im Gegensatz zu den Methoden auf Grundlage technischer
Sammler werden mit Moos-Monitoring auch rezeptorabhängige Einträge erfasst. Es können damit sowohl die feuchte Deposition (Nebel, Tau, Reif) als auch Anteile der trockenen Deposition (Sedimentation und Trägheitsabscheidung durch Filterwirkung, diffusiver Eintrag), die sich weder mit wet only-Sammlern noch mit bulk-Sammlern messen lassen, abgebildet werden. Die ermittelten Werte gelten für Rezeptoren mit ähnlicher Struktur und Oberfläche.

\section{Schlussfolgerungen}

Der mit dem Critical Loads-Ansatz verfolgte Weg, PEC / PNEC-Verhältnisse für atmosphärische N-Einträge auf Ökosystemebene räumlich differenzierend zu berechnen, ist sinnvoll. Er sollte aufgrund einiger Probleme mit den Inputdaten für die Modellierung - Depositionsmessungen aus unterschiedlichen Messprogrammen in Deutschland, deren Designs in räumlicher und zeitlicher Hinsicht sowie in Bezug auf die gemessenen Komponenten und Messmethoden nicht hinreichend harmonisiert sind, Schwierigkeiten beim Zusammenführen der Daten für die Depositionsmodellierung sowie Probleme mit der räumlichen Dichte der Depositionsmessnetze - ergänzt werden durch die Nutzung der Daten über die NAnreicherung infolge atmosphärischer Deposition. Dass sich diese Schwierigkeiten mindern lassen, wenn man die $\mathrm{N}$-Bioakkumulation als physikalisch und chemisch mit ihrer atmosphärischen Deposition gekoppelten als Hilfsvariable bei der Berechnung der N-Deposition verwendet, wurde in dieser Arbeit mit positivem Ergebnis geprüft.

Die Modellierung der atmosphärischen N-Depositionen in Deutschland sollte dem Vorbild der Kooperation zwischen EMEP-Depositionsmodellierung und ICP Vegetation Moos-Monitoring folgen und die Ergebnisse der Depositionsmodellierung durch Koppelung mit den Daten aus dem Moos-Monitoring validieren. Hierzu wäre es zweckmäßig, die Depositionsdatenbank mit dem WebGIS MossMet [61] zu verknüpfen. Dabei wären auch die Informationen, mit denen die Standorte der Depositionsmessungen und die Messungen selbst beschrieben werden, einzupflegen. Solche Metadaten sind für die Moos-Monitoringkampagnen dokumentiert und werden in die statistischen Analysen einbezogen.

\section{Competing interests}

The authors declare that they have no competing interests.

\section{Authors' contributions}

WS wrote the text. MH and RP conducted the computations. $\mathrm{HF}$ and $\mathrm{HH}$ supported the work by dealing with the validity of experimental and modelling data.

\section{Danksagung}

Wir danken dem United Kingdom Department for Environment, Food and Rural Affairs (Defra; contract AQ0810, LEP 0901), der UNECE (Trust Fund) und 
dem Natural Environment Research Council (NERC) für die Finanzierung des Koordinationszentrums des ICP Vegetation Programms am Centre for Ecology and Hydrology (CEH) Bangor, UK. Ferner danken wir den Institutionen für die Bereitstellung der Ergebnisse der nationaler Moos-Monitoring-Kampagnen aus Italien (Renate Alber, Environmental Agency of Bolzano, Laives, Italien), der Türkei (Mahmut Coşkun, Canakkale Onsekiz Mart University, Faculty of Medicine Department of Medical Biology, Çanakkale), Belgien (Ludwig De Temmerman, Veterinary and Agrochemical Research Centre, Tervuren), Lettland (Marina Frolova, Latvian Environment, Geology and Meteorology Agency, Riga), Spanien (Laura González-Miqueo and Jesús M. Santamaría, Departmento de Quimica y Edafologia, Universidad de Navarra, Navarra), Slowenien (Zvonka Jeran, Department of Environmental Sciences, Institut Jozef Stefan; Primož Simonèiè Slovenian Forestry Institute), Finnland (Eero Kubin and Juha Piispanen, Finnish Forest Research Institute, Muhos Research Station, Muhos), Frankreich (Sébastien Leblond, Muséum National d'Histoire Naturelle, Paris), Estland (Siiri Liiv, Tallinn Botanic Garden, Tallinn), der Slowake (Blanka Maňkovská, Institute of Landscape Ecology, Slovak Academy of Science, Bratislava), Tschechien (Ivan Suchara, Silva Tarouca Research Institute for Landscape and Ornamental Gardening), Bulgarien (Lilyana Yurukova, Institute of Botany, Bulgarian Academy of Sciences, Sofia), der Schweiz (Lotti Thöni, FUB - Research Group for Environmental Monitoring, Rapperswil) und Österreich (Harald G. Zechmeister, Faculty of Life Sciences, University of Vienna, Vienna).

\section{Author details}

'Chair of Landscape Ecology, University of Vechta, P.O.B. 1553, 49364 Vechta, Germany. ${ }^{2}$ Centre for Ecology \& Hydrology, Environment Centre Wales, Deiniol Road, Bangor, Gwynedd, LL57 2UW, UK. ${ }^{3}$ Meteorological Synthesizing CentreWest of EMEP, The Norwegian Meteorological Institute, P.O.Box 43-Blindern, N-0313 Oslo, Norway.

Received: 13 April 2011 Accepted: 18 May 2011

Published: 18 May 2011

\section{Zitierte Literatur}

1. Bragazza L, Limpens J, Gerdol R, Grosvernier P, Hájek M, Hájek T, Hajkova P, Hansen I, lacumin P, Kutnar L, Rydin H, Tahvanainen T: Nitrogen concentration and $\delta 15 \mathrm{~N}$ signature of ombrotrophic Sphagnum mosses at different $\mathrm{N}$ deposition levels in Europe. Global Change Biol 2005, 11:106-114.

2. Jovan S, Carlberg T: Nitrogen content of Letharia vulpina tissue from forests of the Sierra Nevada, California: Geographic patterns and relationships to ammonia estimates and climate. Environ Monit Assess 2007, 129:243-251.

3. Slanina S, Wayne D: Air pollution emissions. In Encyclopedia of Earth. First published in the Encyclopedia of Earth October 18, 2006; Last revised August 21, 2008. Edited by Cleveland CJ. [http://www.eoearth.org/article/Air_ pollution_emissions]. Accessed 21 August 2009.

4. Bobbink R, Hicks K, Galloway JN, Spranger T, Alkemade R, Ashmore M, Bustamante M, Cinderby S, Davidson E, Dentener F, Emmett B, Erisman JW, Fenn M, Gilliam F, Nordin A, Pardo L, De Vries W: Global assessment of nitrogen deposition effects on terrestrial plant diversity: a synthesis. Ecol Appl 2010, 20:30-59

5. Erisman JW, de Vries W: Nitrogen deposition and effects on European forests. Environ Rev 2000, 8:65-93.

6. Galloway JN, Townsend AR, Erisman JW, Bekunda M, Cai Z, Freney JR, Martinelli LA, Seitzinger SP, Sutton MA: Transformation of the nitrogen cycle: Recent trends, questions, and potential solutions. Science 2008, 320:889-892.

7. Gundersen P, Rasmussen L: Nitrification in forest soils: Effects from nitrogen deposition on soil acidification and aluminium release. Rev Environ Contam Toxicol 1990, 113:1-45.

8. Pitcairn C, Leith I, Sheppard L, Sutton M, Fowler D, Munro R, Tang S, Wilson D: The relationship between nitrogen deposition species composition and foliar nitrogen concentrations in woodland flora. Environ Pollut 1998, 102 (S1):41-48.

9. Doyle U, Heiß C: Bewertung von diffusen Stoffeinträgen im Rahmen der nationalen Biodiversitätsstrategie. Renaissance der integrierten Umweltbeobachtung. Umweltwiss Schadst Forsch 2009, 21:539-548.

10. Simpson D, Fagerli H, Hellsten S, Knulst JC, Westling O: Comparison of modelled and monitored deposition fluxes of sulphur and nitrogen to
ICP-forest sites in Europe. Biogeosci 2006, 3:337-355.

11. Simpson D, Butterbach-Bahl K, Fagerli H, Kesik M, Skiba U, Tang S: Deposition and emissions of reactive nitrogen over European forests: A modelling study. Atmos Environ 2006, 40:5712-5726.

12. Harmens $\mathrm{H}$, Norris D and the participants of the moss survey: Spatial and temporal trends in heavy metal accumulation in mosses in Europe (1990-2005). Programme Coordination Centre for the ICP Vegetation, Centre for Ecology and Hydrology, Bangor, UK; 2008. [http://icpvegetation.ceh.ac.uk]. Accessed 18 November 2009

13. Harmens H, Norris D, Cooper D, Hall J and the Participants of the Moss Survey: Spatial Trends in Nitrogen Concentrations in Mosses Across Europe in 2005/2006. Programme Coordination Centre for the ICP Vegetation. Centre for Ecology and Hydrology, Environment Centre Wales, Bangor, UK; 2008. [http://icpvegetation.ceh.ac.uk]. Accessed 18 November 2009.

14. Harmens H, Norris DA, Steinnes E, Kubin E, Piispanen J, Alber R, Aleksiayenak Y, Blum O, Coşkun M, Dam M, De Temmerman L. Fernández, JA, Frolova M, Frontasyeva M, González-Miqueo L, Grodzińska K, Jeran Z, Korzekwa S, Krmar M, Kvietkus K, Leblond S, Liiv S, Magnússon SH, Maňkovská B, Pesch R, Rühling Å, Santamaria JM, Schröder W, Spiric Z, Suchara I, Thöni L, Urumov V, Yurukova L, Zechmeister HG: Mosses as biomonitors of atmospheric heavy metal deposition: spatial and temporal trends in Europe. Environ Pollut 2010, 158: 3144-3156.

15. Harmens H, Mills G, Hayes F, Williams P, De Temmerman L and the participants of ICP Vegetation: Air pollution and vegetation. ICP Vegetation Annual Report 2004/2005. UNECE ICP Vegetation Coordination Centre, Centre for Ecology and Hydrology, Bangor, UK; 2005. [http://icpvegetation.ceh.ac.uk]. Accessed 18 November 2009

16. Pitcairn CER, Fowler D, Grace J: Deposition of fixed atmospheric nitrogen and foliar nitrogen content of bryophytes and Calluna vulgaris (L.) Hull. Environ Pollut 1995, 99:193-205.

17. Pitcairn C, Fowler D, Leith I, Sheppard L, Tang S, Sutton M, Famulari D: Diagnostic indicators of elevated nitrogen deposition. Environ Pollut 2006, 144:941-950.

18. Poikolainen J, Piispanen J, Karhu J, Kubin E: Long-term changes in nitrogen deposition in Finland (1990-2006) monitored using the moss Hylocomium splendens. Environ Pollut 2009, 157:3091-3097.

19. Salemaa M, Mäkipää R, Oksanen J: Differences in the growth response of three bryophyte species to nitrogen. Environ Pollut 2008, 152:82-91.

20. Solga A, Burkhardt J, Zechmeister HG, Frahm J-P: Nitrogen content, 15N natural abundance and biomass of the two pleurocarpous mosses Pleurozium schreberi (Brid.) Mitt., and Scleropodium purum (Hedw.) Limpr. in relation to atmospheric nitrogen deposition. Environ Pollut 2005, 134:465-473.

21. Zechmeister HG, Hohenwallner D, Smidt S, Roder I, Maringer S, Richter A, Wanek W: Total nitrogen content and $\delta 15 \mathrm{~N}$ signatures in moss tissue: Indicative value for nitrogen deposition patterns and source allocation on a nation-wide scale. Environ Sci Technol 2008, 42:8661-8667.

22. Lorenz M, Granke O: Deposition measurements and critical loads calculations: monitoring data, results and perspective. iForest 2009, 2:11-14

23. Spranger T, Kunze F, Gauger T, Nagel D, Bleeker A, Draaijers G: Critical loads exceedances in Germany and their dependence on the scale of input data. Water Air Soil Pollut 2001 (Focus 1):335-351.

24. Schröder W, Pesch R, Matter Y, Göritz A, Dieffenbach-Fries H, Genßler L: Trend der Schwermetall-Bioakkumulation 1990 bis 2005: Qualitätssicherung bei Probenahme, Analytik, geostatistischer Auswertung. Umweltwiss Schadst Forsch 2009, 21:549-574.

25. Ferretti M: Forest health assessment and monitoring - Issues for consideration. Environ Monit Assess 1997, 48:45-72.

26. Ferretti M, König N, Rautio P, Sase H: Quality assurance (QA) in international forest monitoring programmes: activity, problems and perspectives from East Asia and Europe. Ann For Sci 2009, 66:403/1-403/12

27. Wuyts K, De Schrijver A, Verheyen K: The importance of forest type when incorporating forest edge deposition in the evaluation of critical load exceedance. iForest 2009, 2: 43-45.

28. Gauger T, Haenel H-D, Rösemann C, Dämmgen U, Bleeker A, Erisman JW Vermeulen AT, Schaap M, Timmermanns RMA, Builtjes PJH, Duyzer JH: National Implementation of the UNECE Convention on Long-range Transboundary Air Pollution (Effects) Part 1: Deposition Loads: Methods, modelling and mapping results, trends. UBA-Texte 38/08. Dessau; 2008.

29. Gauger T, Haenel H-D, Rösemann C, Nagel H-D, Becker R, Kraft P, Schlutow A, 
Schütze G, Weigelt-Kirchner R, Anshelm F: Nationale Umsetzung der EU-NECRichtlinie/CAFE-Strategie und UNECE Luftreinhaltekonvention. Teil 2: Wirkungen und Risiokoabschätzungen, Critical Loads, Biodiversität, Dynamische Modellierung, Critical Levels Überschreitungen, Materialkorrosion. UBA-Texte 38/08. Dessau, 2008.

30. UBA (Umweltbundesamt): Workshop zur Modellierung von Schadstoffeinträgen und ihren Wirkungen auf Ökosysteme am 8.12.2009 im Umweltbundesamt. Dessau, 2010. [http://www.umweltbundesamt.de/luft/infos/veranstaltungen/ ws_mapesi.htm]. Accessed 26 January 2010.

31. ICP Vegetation: Heavy metals in European mosses: 2005/2006 survey. Monitoring manual. ICP Vegetation Coordination Centre, Centre for Ecology and Hydrology, Bangor, UK; 2005. [http://icpvegetation.ceh.ac.uk]. Accessed 26 January 2010.

32. Pesch R, Schröder W, Dieffenbach-Fries H, Genßler L, Kleppin L: Optimierung des Moosmonitoring-Messnetzes in Deutschland. Umweltwiss Schadst Forsch 2008, 20:49-61.

33. Kjeldahl J: A new method for the determination of nitrogen in organic matter. ZAnal Chem 1883, 22:366.

34. Dumas JBA: Procédes de I'Analyse Organique. Ann Chim Phys 1831, 247:198-213

35. O'Connor P: Monitoring - Quality assurance and quality control. Government of South Australia. South Australian Murray-Darlin Basin. Natural Resources Management Board; 2009. [http://www.samdbnrm.sa.gov. au/Portals/7/Monitoring\%20Quality\%20Assurance\%20and\%20Quality\%20 Control\%20(C).pdf]. Accessed 25 November 2009.

36. Mohr K, Holy M, Pesch R, Schröder W: Bioakkumulation von Metallen und Stickstoff zwischen 1990 und 2005 in Niedersachsen. Umweltwiss Schadst Forsch 2009, 21:459-464.

37. Steinnes E, Rühling Å, Lippo H, Mäkinen A: Reference materials for largescale metal deposition surveys. Accredit Qual Assur, 2:243-249.

38. Berge $E$, Jakobsen HA: A regional scale multi-layer model for the calculation of long-term transport and deposition of air pollution in Europe. Tellus 1998, 50:205-223.

39. Jonson JE, Bartnicki J, Olendrzynski K, Jakobsen HA, Berge E: EMEP Eulerian model for atmospheric transport and deposition of nitrogen species over Europe. Environ Pollut 102:289-298.

40. Fagerli H, Simpson D, Tsyro S: Transboundary acidification, eutrophication and ground level ozone in Europe. EMEP Status Report 1/2004, Unified EMEP model. The Norwegian Meteorological Institute, Oslo, Norway, Updates; 2004:11-18

41. Simpson D, Fagerli H, Jonson JE, Tsyro S, Wind P, Tuovinen J-P: Transboundary acidification and eutrophication and ground level ozone in Europe: Unified EMEP Model Description. EMEP Status Report 1/2003 Part I, EMEP/MSC-W Report. The Norwegian Meteorological Institute, Oslo, Norway; 2003.

42. Fagerli H, Aas W: Trends of nitrogen in air and precipitation: Model results and observations at EMEP sites in Europe, 1980-2003. Environ Pollut 154:448-461.

43. Fagerli $H$, Simpson D, Aas W: Model performance for sulphur and nitrogen compounds for the period 1980 to 2000. In Transboundary Acidification, Eutrophication and Ground Level Ozone in Europe. EMEP Status Report 1/2003, Part II Unified EMEP Model Performance. Edited by Tarrasón L. The Norwegian Meteorological Institute, Oslo, Norway: 2003:1-66.

44. Fagerli $H$, Legrand M, Preunkert S, Simpson D, Vestreng V, Cerqueira, M: Modeling historical long-term trends of sulfate, ammonium and elemental carbon over Europe: A comparison with ice core records in the Alps. J Geophys Res 2007, 112:D23S13.

45. Jonson JE, Simpson D, Fagerli $\mathrm{H}$, Solberg S: Can we explain the trends in European ozone levels? Atmos Chem Phys 2006, 6:51-66.

46. Simpson D, Yttri KE, Klimont Z, Kupiainen K, Caseiro A, Gelencsér A, Pio C, Legrand M: Modeling carbonaceous aerosol over Europe. Analysis of the CARBOSOL and EMEP EC/OC campaigns. J Geophys Res 2007, 112:D23S14.

47. Tsyro S, Simpson D, Tarrasón L, Klimont Z, Kupiainen K, Pio C, Yttri KE: Modeling of elemental carbon over Europe. J Geophys Res 2007, 112:D23S19.
48. Vestreng V, Adams M, Goodwin J: Inventory review 2004. Emission data reported to CLRTAP and under the NEC directive. EMEP/EEA Joint Review Report, Tech. Rep. EMEP-MSCWReport 1/2004. The Norwegian Meteorological Institute, Oslo, Norway; 2004

49. Gauger T: Nationale Luftreinhaltestrategie - Umsetzung von EU-Anforderungen; Teilvorhaben 02: Aufbereitung, Nutzung und Weiterentwicklung nationaler, hochauflösender Datensätze zu Konzentrationen und Depositionen von Luftschadstoffen. Forschungsvorhaben im Auftrag des BMU/UBA, FE-Nr. 203 43 257/02. 23 S. Text + 54 S. Anhang. FAL-AOE, Braunschweig; 2005.

50. Gauger T, Anshelm F: Mapping of ecosystem specific long-term trends in deposition loads and concentrations of air pollutants in Germany and their comparison with Critical Loads and Critical Levels. Part 2: Mapping Critical Levels exceedances. Research Project 29942210 on behalf of Federal Environmental Agency. Final Report. Berlin, Stuttgart; 2002.

51. Gauger T, Anshelm F, Köble R: Kritische Luftschadstoff-Konzentrationen und Eintragsraten sowie ihre Überschreitung für Wald und Agrarökosysteme sowie naturnahe waldfreie Ökosysteme. Forschungsvorhaben im Auftrag des BMU / UBA, FE-Nr. 29785 079. Institut für Navigation, Universität Stuttgart. Teil 1: Deposition Loads. Berlin, Stuttgart; 2000.

52. Gauger T, Anshelm F, Köble R: Kritische Luftschadstoff-Konzentrationen und Eintragsraten sowie ihre Überschreitung für Wald und Agrarökosysteme sowie naturnahe waldfreie Ökosysteme. Forschungsvorhaben im Auftrag des BMU/ UBA, FE-Nr. 29785 079. Institut für Navigation, Universität Stuttgart. Teil - Teil 2: Critical Levels. Berlin, Stuttgart; 2000.

53. Gauger T, Anshelm F, Schuster H, Draaijers GPJ, Bleeker A, Erisman JW, Vermeulen AT, Nagel HD: Mapping of ecosystem specific long-term trends in deposition loads and concentrations of air pollutants in Germany and their comparison with Critical Loads and Critical Levels. Part 1: Deposition Loads 19901999. Research Project 29942210 on behalf of Federal Environmental Agency. Final Report. Berlin, Stuttgart; 2002

54. Schaub M: Future monitoring and research needs for forest ecosystems in a changing environment: an introduction. iForest, 2009 2:54-55.

55. Bertino L,Wackernagel H: Case studies of change-of-support problems. Technical report N-21/02/G, ENSMP_ARMINES. Centre de Géostatistique, Fontainebleau, France; 2002

56. Genikhovich E, Filatova E, Ziv A: A method for mapping the air pollution in cities with the combined use of measured and calculated concentrations. Int J Environ Pollut 2002, 18:56-63.

57. Goovaerts P: Geostatistical approaches for incorporating elevation into the spatial interpolation of rainfall. J Hydrol 2000, 228:113-129.

58. Pauly M, Drueke M: Mesoscale spatial modelling of ozone immissions. An application of geostatistical methods using a digital elevation model. Gefahrstoffe-Reinhalt Luft 1996, 56:225-230.

59. Van de Kassteele J, Stein A, Dekkers ALM, Velders GJM: External drift kriging of NOx concentrations with dispersion model output in a reduced air quality monitoring network. Environ Ecol Stat 2009, 16:321-339.

60. Knappe F, Möhler S, Ostermayer A, Lazar S, Kaufmann C: Vergleichende Auswertung von Stoffeinträgen in Böden über verschiedene Eintragspfade. UBA-Texte 36/08. Dessau, 2008.

61. Kleppin L, Schröder W, Pesch R, Schmidt G: Entwicklung und Erprobung einer Metadaten- und WebGIS-Applikation für das Expositionsmonitoring mit Moosen in Deutschland. Ein Beitrag zum LTER-Netzwerk. Umweltwiss Schadst Forsch 2008, 20:38-48.

doi:10.1186/2190-4715-23-18

Cite this article as: Schröder W, et al:: Mapping background values of atmospheric nitrogen total depositions in Germany based on EMEP deposition modelling and the European Moss Survey 2005. Environmental Sciences Europe 2011, 23:18 